\title{
BMJ Open Effectiveness of an antenatal maternal supplementation with prebiotics for preventing atopic dermatitis in high- risk children (the PREGRALL study): protocol for a randomised controlled trial
}

Clémentine Cabridain, ${ }^{1}$ Hélène Aubert, ${ }^{2}$ Bertrand Kaeffer, ${ }^{3}$ Virginie Badon, ${ }^{4}$ Marion Boivin, ${ }^{4}$ Vincent Dochez, ${ }^{4,5}$ Norbert Winer, ${ }^{4,5}$ Elodie Faurel-Paul, ${ }^{6}$ Lucie Planche, ${ }^{7}$ David Riochet, ${ }^{8}$ Annabel Maruani, ${ }^{9,10}$ Franck Perrotin, ${ }^{11,12}$ Catherine Droitcourt, ${ }^{13,14}$ Linda Lassel, ${ }^{15}$ Martine Tching-Sin, ${ }^{16}$ Natasha K Rogers, ${ }^{17}$ Marie Bodinier, ${ }^{18}$ Sebastien Barbarot ${ }^{19}$

To cite: Cabridain C, Aubert H, Kaeffer B, et al. Effectiveness of an antenatal maternal supplementation with prebiotics for preventing atopic dermatitis in high-risk children (the PREGRALL study): protocol for a randomised controlled trial. BMJ Open 2019;9:e024974. doi:10.1136/ bmjopen-2018-024974

- Prepublication history and additional material for this paper are available online. To view these files, please visit the journal online (http://dx.doi. org/10.1136/bmjopen-2018024974).

$\mathrm{CC}$ and $\mathrm{HA}$ contributed equally. $\mathrm{MB}$ and SB contributed equally.

Received 25 June 2018 Revised 6 February 2019 Accepted 12 February 2019

\section{Check for updates}

(c) Author(s) (or their employer(s)) 2019. Re-use permitted under CC BY-NC. No commercial re-use. See rights and permissions. Published by BMJ.

For numbered affiliations see end of article.

Correspondence to Dr Sebastien Barbarot; sebastien.barbarot@chunantes.fr

\section{ABSTRACT}

Introduction Atopic dermatitis (AD) is a chronic inflammatory disease affecting $10 \%-15 \%$ of children in Europe. There is a need for new primary preventive therapeutic strategies in at-risk populations. Recent research has indicated that atopic diseases are associated with a disrupted gut microbial 'balance' in early life raising the possibility that interventions which yield optimal patterns of microflora could improve host's health. Prebiotics, sugars with immunomodulatory properties that stimulate the diversity of the digestive microbiota, are ideal candidates for such research. So far, most clinical trials have focused on improving infant gut colonisation postnatally. However, prenatal life is a crucial period during which different tolerance mechanisms are put in place. We aim to determine whether antenatal prebiotics supplementation prevents $A D$ in high-risk children. Methods and analysis This is a randomised, multicentre, double-blind, trial to evaluate the effectiveness of antenatal prebiotic maternal supplementation (galactooligosaccharide/inulin) in pregnant women versus placebo on the occurrence of $A D$ at 1 year of age in at-risk children (defined as having a maternal history of atopic disease). Participating women will be randomised to daily ingestion of a prebiotics or placebo (maltodextrin) from 20 weeks' gestation until delivery. The primary outcome is the prevalence of $A D$ at 1 year of age, using the version of the UK Working Party Diagnostic Criteria optimised for preventive studies. Key secondary endpoints are AD severity, quality of life and prebiotics tolerance. The target sample size is 376 women (188 patients per group) which will provide $80 \%$ power to detect a $33 \%$ reduction of the risk of $A D$ in the verum group ( $\alpha=0.05)$. The primary analysis will be based on the intention-to-treat principle. Ethics and dissemination Results will be presented in peer-reviewed journals and at international conferences. Ethics approval for the study was obtained from the institutional ethical review board of 'Comité de Protection

\section{Strengths and limitations of this study}

This study uses an innovative prevention strategy as it will be the first clinical trial assessing the effects of prebiotics exclusively in pregnancy for atopic dermatitis prevention in high-risk children.

- A feasibility study was conducted to assess the recruitment capacities of the centres involved.

- An ancillary study will use biological samples from 50 infant-mother dyads ( 25 per group) to evaluate underlying mechanisms.

- The results of this study may not be generalised in different populations due to selection bias.

des Personnes Sud Ouest-Outre-Mer III' of the University Hospital Centre of Bordeaux (2017/13).

Trial registration number NCT03183440; Pre-results.

\section{INTRODUCTION}

Allergies affect $30 \%-40 \%$ of the world's population, with their increasing prevalence recognised as a significant public health issue. $^{1}$

Allergies arise from an abnormal reaction of the immune system (IS) to an allergen, with the production of antigen-specific IgE. The term 'atopic' describes individuals with a predisposition to mount inappropriate $\mathrm{IgE}$ responses. Atopic dermatitis (AD) is the earliest and the most common manifestation of allergic disease (prevalence 10\%-15\% ${ }^{2}$ ), and is thought to be the first step of the 'atopic march', a term used to describes the tendency for allergic diseases to develop sequentially 
throughout childhood. AD is a chronic condition; $40 \%$ of children who present with $\mathrm{AD}$ have symptoms which persistent into adulthood, ${ }^{3}$ incurring considerable care and social costs. As a highly prevalent disease with no cure, primary prevention strategies for $\mathrm{AD}$ are essential, and may herald a way to circumvent the atopic march.

Recent research has indicated that allergies are associated with a disrupted gut microbial balance, and a link between IS deregulation and decreased diversity of neonates' gut microbiota has been identified. ${ }^{45}$ This raises the possibility that interventions which restore the normal balance of microflora could improve health. Prenatal life constitutes the first period where the immune and microbial systems of the fetus can be influenced, with immune factors and bacteria transfers through the cord blood in utero. ${ }^{67}$ As maternal exposure to environmental factors influences the development of these systems in her offspring, pregnancy offers a unique window of opportunity for an intervention to modulate the immune and microbial systems of the fetus.

Prebiotics (commonly galacto-oligosaccharide and/or fructo-oligosaccharide [GOS/FOS]) are non-digestible food components that can promote the growth of certain bacteria in the gut. Our previous studies with murine models demonstrated that prebiotic supplementation during the perinatal period is associated with reinforced gut barrier functions ${ }^{8}$, tolerance induction ${ }^{9}$ and reduced allergic reactions. ${ }^{10}$ This line of enquiry has been investigated by other research teams, and has demonstrated that prebiotics supplementation during pregnancy reduced eczema-like inflammation $^{11}$ and respiratory allergy $^{12}$ in mice offspring.

To date, clinical trials have been limited to postnatal prebiotics supplementation. For infants with a low risk of atopy, receiving diets supplemented with prebiotics (GOS/inulin/pectin), a preventive effect on $\mathrm{AD}$ was observed 1year of age ${ }^{13}$ but not at 5 years. ${ }^{14}$ A recent systematic review and meta-analysis found a significant reduction in $\mathrm{AD}$ (risk ratio: $0.68,95 \%$ CI 0.40 to 1.15 ) ${ }^{15}$ The authors of a 2013 Cochrane review concluded that there was some evidence that a prebiotic supplement added to infant feeds may prevent $\mathrm{AD}$ but further research is still needed before the routine use of prebiotics can be recommended for the prevention of atopic diseases. ${ }^{16}$

Based on our preclinical results, we assume that an earlier intervention during pregnancy, when fetal responses are first developed, is likely to be more effective than a late or postnatal intervention. Our study will therefore test the hypothesis that maternal antenatal prebiotics (GOS/inulin) supplementation is superior to placebo for $\mathrm{AD}$ prevention in high-risk children (PREGRALL study).

\section{METHODS AND ANALYSIS}

This protocol adheres to the Standard Protocol Items: Recommendations for Interventional Trials recommendations for interventional trials.

\section{Trial design}

The PREGRALL trial is a randomised, placebo-controlled, double-blind, multicentre trial with two parallel groups.

\section{Participating centres}

The four participating centres, Nantes University hospital, Tours University Hospital, Angers University Hospital and Rennes University Hospital, are university hospitals located in the West region of France and are all part of the Hôpitaux Universitaires du Grand Ouest PEdiatric REsearch Network network.

\section{Eligibility criteria}

Pregnant women will be approached by trained clinical trial personnel in the antenatal clinic of participating centres.

\section{Inclusion criteria}

- Pregnant women ( $\geq 18$ years old) 20-week gestation $( \pm 6$ days $)$.

- In attendance of an antenatal clinic of a participating centre.

- Maternal history of atopy (defined as a healthcare professional diagnosis of asthma, allergic rhinitis, atopic eczema or food allergy).

- Able to give informed consent.

\section{Exclusion criteria}

- Women unwilling to stop taking dietary supplements (including prebiotics or probiotics) for the duration of the supplementation period.

- Any apparent fetal or maternal pathology diagnosed before 20 weeks. Maternal pathologies include, but not limited to, pre-eclampsia, high blood pressure, known autoimmune disease, infectious pathology, uterine malformation, cervical incompetence or insufficiency requiring cervical cerclage, and gestational diabetes. Fetal pathologies include, but are not limited to, intrauterine fetal growth restriction or small weight for gestational age, fetal malformation oligohydramnios and polyhydramnios.

- Maternal smoking during pregnancy.

- Women allergic to cow's milk protein and/or lactose intolerance owing to the lactose content of GOS.

- Any other condition which would, in the investigator's opinion, deem the patient unsuitable for participation in the study (eg, psychosocial problems).

\section{Study treatments}

Participating women will be randomised to a powdered preparation of either prebiotics (GOS/inulin) or placebo (maltodextrin). A single daily dose is prepared by diluting two standardised measuring spoons of powder in fluid at room temperature. Women will be asked to consume the preparation every morning from the 20th week of gestation ( \pm 6 days) until delivery. 
GOS/inulin mixture

The GOS (DOMO VIVINAL GOS powder, FrieslandCampina, The Netherlands) and inulin (Orafti HP, BENEO-Orafti, Belgium) is mixed in a 9:1 ratio. This ratio strikes a compromise between tolerability and efficiency according to the literature. ${ }^{17}$ In order to obtain $11.8 \mathrm{~g}$ of GOS/inulin powder per day per woman, a mixture of $8.36 \mathrm{~g}$ pure GOS (10.91 g GOS powder) and $0.83 \mathrm{~g}$ pure inulin ( $0.86 \mathrm{~g}$ inulin powder) will be prepared.

It should be noted that this is an independent trial; the manufacturers of the products used have not been involved in the planning or funding of the trial, nor will they be involved in the conduct or analysis.

\section{Placebo}

Maltodextrin is a relatively inert raw material composed of carbohydrates. Provided in a powder form, it can be diluted in a liquid at room temperature. To be isocaloric with the GOS/inulin mixture $12.4 \mathrm{~g}$ will be prepared per day for ingestion.

\section{Manufacture of study powders}

The study powders will be packaged in identical boxes in the pharmacy of the coordinating centre according to the code of Good Manufacturing Practice (GMP). The powders are packaged and labelled in accordance with GMP including an individual product identifier (ID), batch number and expiry date. The pharmacy at the coordinating centre will supply the participating centres pharmacies with the study powders. Study powders will be dispensed at 20-week gestation (four boxes supplied) and again at 32-week gestation (two boxes supplied).

\section{Monitoring adherence to study treatment}

Research personnel at each centre will maintain regular contact with participants to monitor and encourage treatment adherence, as well as to answer any questions as they arise. In addition, participants are asked to note any omissions in a diary which we provided to them (from 0 to 7 omissions per week). Adherence will be assessed by capturing the number of omission at each visit. Women are asked to return unused boxes at delivery.

\section{Outcomes measures}

\section{Primary outcome}

The primary outcome is the prevalence of $\mathrm{AD}$ at 1 year of age, using the version of the UK Working Party Diagnostic Criteria optimised for preventive studies ${ }^{18}$ as follows.

A history of an itchy skin condition that is either continuous or intermittent lasting at least 4 weeks plus 3 or more of the following:

1. A history of a rash in the skin creases (folds of elbows, behind the knees, fronts of ankles or around the neck) or on the extensor aspects of the forearms or lower legs.

2. A personal history of asthma or hay fever or a history of atopic disease in a first-degree relative.

3. A history of a generally dry skin since birth.
4. Visible flexural dermatitis and/or visible dermatitis on the forearms or lower legs with the absence of axillary involvement.

These criteria exclude transient skin rashes (less than 4 weeks) common in the first year of life in the study population.

\section{Key secondary outcomes}

- The prevalence of $\mathrm{AD}$ at 6 months of age defined according to the UK Working Party Diagnostic Criteria optimised for preventive studies ${ }^{19}$ International Study of Asthma and Allergies in Childhood diagnostic criteria assessed by a telephone questionnaire administered to parents and a healthcare provider diagnosis of $\mathrm{AD}$.

- The severity of $\mathrm{AD}$ at 1 year of age assessed by the Scoring Atopic Dermatitis score ${ }^{20}$ and the Patient-Oriented Eczema Measure. ${ }^{21}$

- The quality of life of the child and their family at 1 year assessed by the Family Dermatitis Quality of Life Index (FDQLI) score. ${ }^{22}$

- Sensitisation status to common allergens as assessed by skin prick tests performed on the child at 1 year old, and parent reported food allergy. Skin prick tests will be applied to the volar aspect of the forearm, at least $2-3 \mathrm{~cm}$ from the wrist and the antecubital fossae. All tests, including the histamine and negative control test results, are read 15-20 min following application. The largest diameter of the weal of each particular test is measured, a positive being a weal of $\geq 3 \mathrm{~mm}^{23}$. A standard skin prick tests panel (ALK or Stallergenes laboratory) for inhalant and food allergens will be tested (milk, egg, peanut, cat, grass pollen, house dust mite).

- Biocollection outcomes. The PREGRALL clinical trial affords an opportunity to evaluate the impact of prebiotic supplementation. Consequently, the ancillary study Characterising the effect of maternal prebiotic supplementation on perinatal IS maturation, Microbiota and breast Milk compositions for Allergy Prevention in high-risk children (CIMMAP) will collect biological samples from 50 infantmother dyads (25 per group). Samples will include blood and stools from both mother and infant; cord blood; colostrum and breastmilk collected at various timepoints (see online supplementary file 1). CIMMAP shall explore the perinatal period in infants at risk of atopy, focusing on the IS, gut microbiota and breastmilk compositions. CIMMAP will help to understand more precisely the mechanics of the clinical effect that could be observed in the PREGRALL trial.

\section{Exploratory secondary outcome}

Transepidermal water loss of infant measured at 2 days and 12 months of age (only relates to the CIMMAP participants). 


\section{Safety outcomes}

The tolerance of prebiotics is good but some minor-tomoderate digestive disorders may occur, and such effects (bloating, stomach aches, diarrhoea, flatulence) will be collected in a diary from inclusion to delivery.

\section{Participant timeline}

Participants will be randomised and commence study treatment from enrolment (20-week gestation, \pm 6 days) until delivery (figure 1). At enrolment, following informed consent, baseline clinical and demographic data will be collected including mother's level of education, number of siblings, living environment (rural or urban). Research personnel will contact the participant 2 weeks following the enrolment visit and again at 24-week and 32-week gestation to ascertain treatment adherence and record any adverse events. At birth, the following outcome will be captured: mode of delivery, gestation (weeks) and birth weight. National Guidelines for Child Dietary Diversification (mangerbouger.fr) will be sent to participants by email or by post at 3 months after delivery. Participants will be called 6 months after delivery. A telephone questionnaire will be administered at 6 months for secondary outcomes. A clinical visit will be carried out 1 year after delivery for primary and secondary outcomes. (See table 1 for a summary of the PREGRALL study.)

\section{Randomisation, allocation concealment and blinding}

The randomisation will be performed by a member of the clinical research team not involved in the study and conducted using a dedicated, password-protected, Secure Sockets Layer (SSL)-encrypted website (Ennov Clinical) and the treatment allocations will remain concealed until database unlock. Patients will be allocated to interventions in a 1:1 ratio using block randomisation stratified by centre and atopy status of the parents (maternal or biparental).

Patients and outcome assessors will be blinded. In order to preserve blinding of the participants, the powdered preparations are isocaloric, identical in colour and taste, and contained within identical packaging.

\section{Sample size}

According to the literature, the risk of $\mathrm{AD}$ at 1 year in children at risk is estimated at $45 \% .{ }^{24}$ We assume a $33 \%$ risk reduction for children whose mothers have been exposed to prebiotics, or a $30 \%$ risk of $\mathrm{AD}$. With $80 \%$ power and $5 \%$ alpha risk, 326 participants are needed. To ensure statistical power, 376 participants (188 per group) will be randomised.

\section{Patient and public involvement}

Patients were not formally involved in the set-up of the trial but our feasibility study explored participants' views of potential involvement, particularly about the burden participation. This was done so that our approach for the main trial could be modified in response to their feedback. Trial participants will receive a short lay summary of the results.

\section{Feasibility study}

We conducted a feasibility study with pregnant women who met the eligibility criteria for the full trial in order to evaluate our proposed recruitment strategy and make any refinements if required (figure 2).

From a list of 350 pregnant women at 20 weeks of gestation in attendance of an obstetrics department of one tertiary hospital, 74 were excluded due to having high-risk pregnancies or psychosocial difficulties deemed incompatible with a clinical trial. A total of 276 women received telephone calls from 7 March 2017 to 26 April 2017 for a pilot-tested semidirective questionnaire evaluating the inclusion and exclusion criteria of the PREGRALL and CIMMAP studies and willingness to participate in the trial. In case of non-response, two further phone calls were made. Women who did not respond after three calls were considered as non-responders. The participants were informed that the purpose of the survey was to gather their views on a future trial, in which they would not be involved. Participants gave their consent orally at the beginning of the questionnaire. Primary outcome was the proportion of women willing to participate in PREGRALL clinical trial among eligible women. The secondary outcomes were (1) the proportion of eligible women meeting the inclusion criteria; (2) the proportion of eligible women willing to participate in the CIMMAP biocollection; (3) the reasons for refusal for PREGRALL and/or CIMMAP studies.

Of the 109 participants included in the feasibility study (1 refusal, 166 were non-responders), 25 (23\%) were potentially eligible for the PREGRALL trial. Of the 25 eligible women, 17 (68\%) declared that they would agree to participate in the PREGRALL trial (primary outcome) and $12(48 \%)$ in the CIMMAP biocollection. Thus, respectively, $15.6 \%(17 / 109)$ and $11 \%(12 / 109)$ of the included participants would agree to participate in the PREGRALL study and the CIMMAP biocollection. Current mothers tended to more likely agree to participate in PREGRALL compared with those expecting their first child. Age and parity did not have a significant effect on willingness to participate in PREGRALL (data not shown). Most participants did not know what prebiotics were. Women without a history of $\mathrm{AD}$ in their first child or with a mild personal history of $\mathrm{AD}$ were less likely to want to participate (data not shown).

The feasibility study confirmed the capacity for inclusion in a centre and models the duration of the trial according to the size of the pregnant women population seen before 20 weeks of gestation in each centre. Using this data and factoring for a $20 \%$ drop-out rate at 1 year, we will have to screen a total of 2897 pregnant women to enrol 376 participants in the PREGRALL multicentre study. Our recruitment models on each of the investigation sites indicate that approximately $50 \%$ of pregnancies taking place in antenatal clinics benefit from obstetrical and/or ultrasound follow-up initiated between 12-week and 24-week gestation. Considering that approximately 3000 pregnant women per year (excluding high-risk 


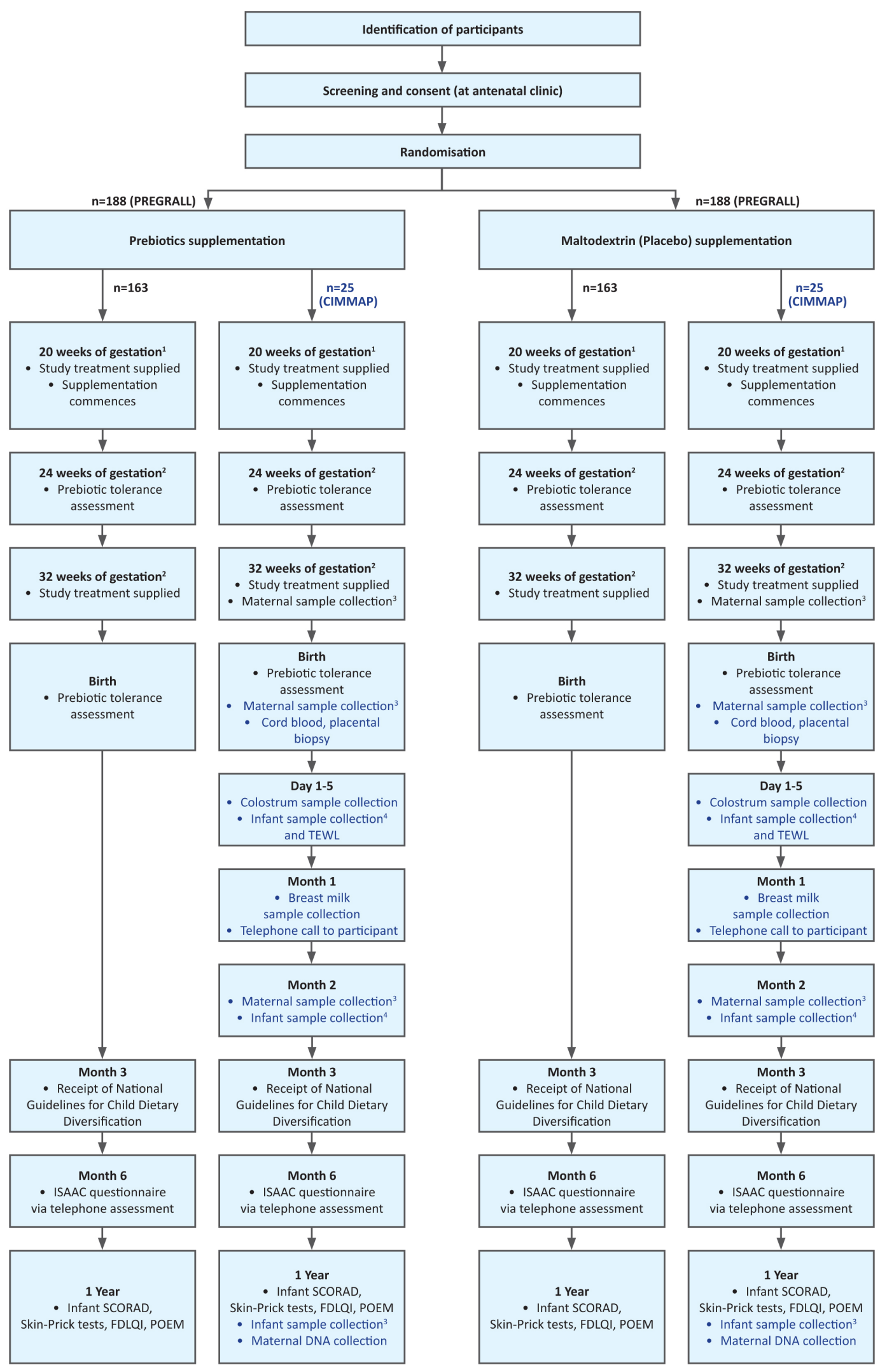

Figure 1 Participant flow diagram. ${ }^{1}$ Timepoints \pm 6 days; ${ }^{2}$ timepoint \pm 7 days; ${ }^{3}$ blood, stool and DNA; ${ }^{4}$ stool and DNA. CIMMAP, Characterising the effect of maternal prebiotic supplementation on perinatal Immune system maturation, Microbiota and breast Milk compositions for Allergy Prevention in high-risk children; FDLQI, Family Dermatitis Quality of Life Index; ISAAC, International Study of Asthma and Allergies in Childhood; POEM, Patient-Oriented Eczema Measure; SCORAD, Scoring Atopic Dermatitis; TEWL, transepidermal water loss. 

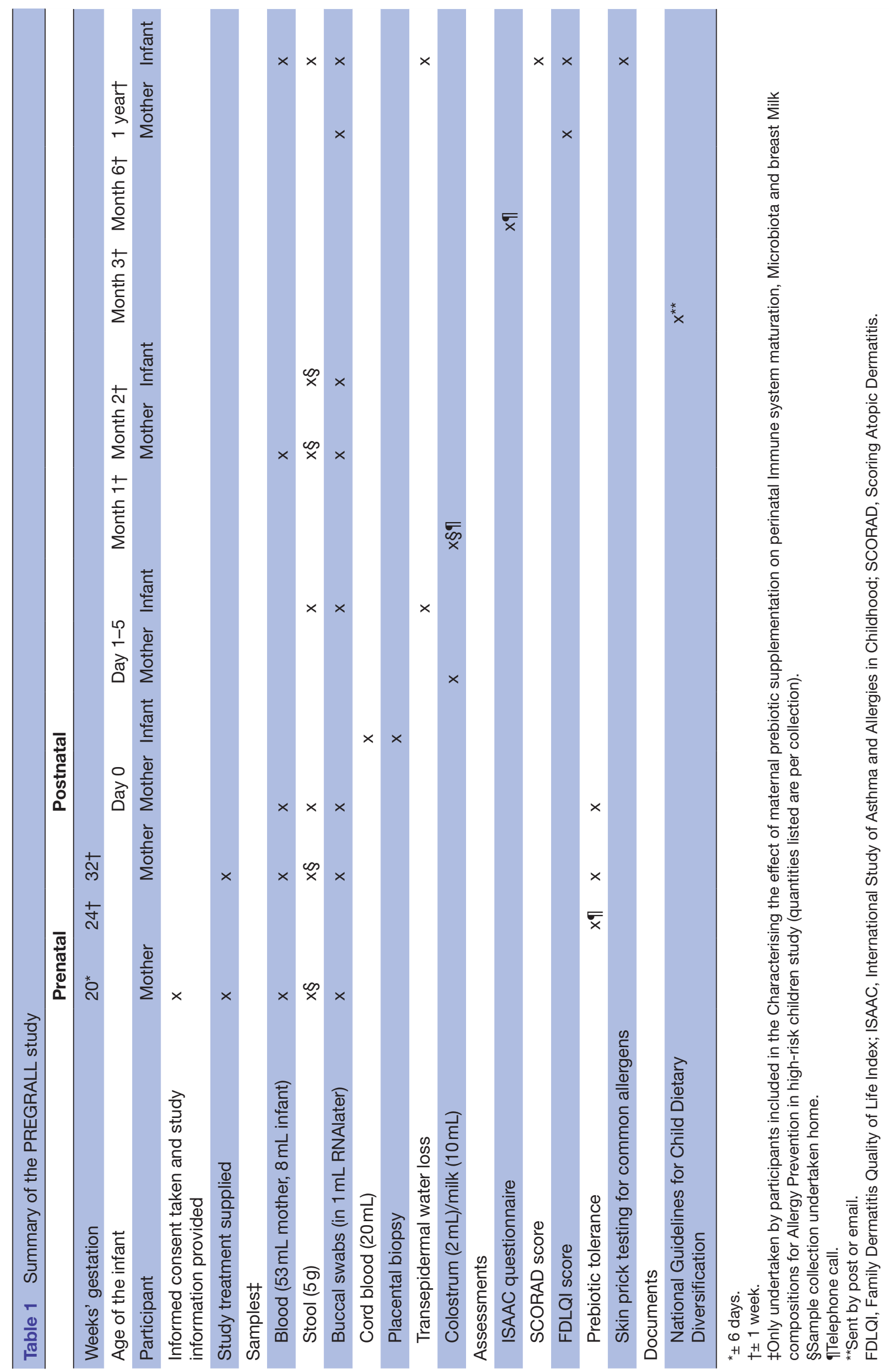

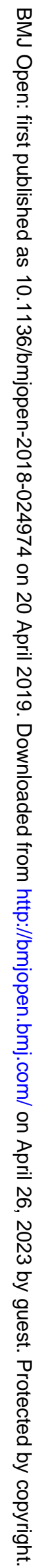




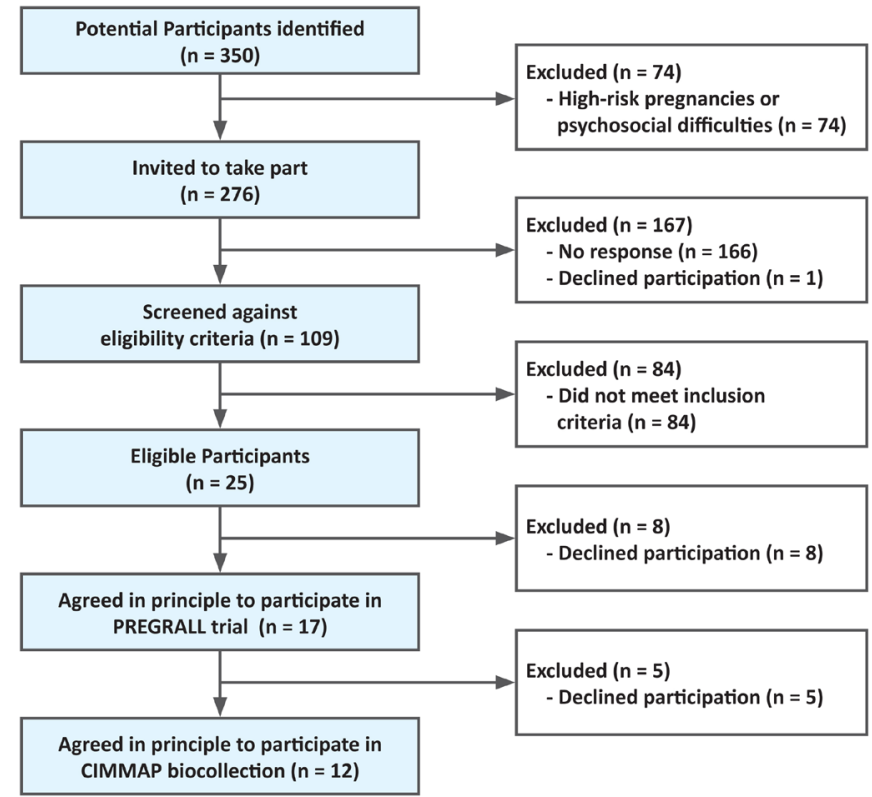

Figure 2 Participant flow in the feasibility study. CIMMAP, Characterising the effect of maternal prebiotic supplementation on perinatal Immune system maturation, Microbiota and breast Milk compositions for Allergy Prevention in high-risk children.

pregnancies) contact our four centres before 20-week gestation, a 24-month period of recruitment seems reasonable. Furthermore, based on these data, the feasibility of the CIMMAP biocollection $(n=100)$ in one centre seems also reasonable. Our study also indicates that providing information to women at the time of selection will increase the likelihood of trial participation. Finally, it shows that stool samples are not a major cause of refusal to participate in the biocollection.

\section{Start and end dates of the study}

The study started on the 13th of February 2018 and will end on the 13th of August 2020.

\section{Statistical methods}

Statistical analyses will be performed using SAS V.9.4 software. The primary analysis will be based on the intention-to-treat principle and will be complemented by a per-protocol analysis.

The primary outcome, occurrence of an $\mathrm{AD}$ at 1year, will be analysed using a generalised linear mixed model adjusted for the stratification variables of parental history of atopy (maternal or biparental) as fixed effect and the centre as random effect. In each group, the occurrence of an $\mathrm{AD}$ at 1 year will be estimated with a $95 \%$ CI. This will be supplemented by an analysis that includes known risk factors for $\mathrm{AD}$, that is, breastfeeding (at least 1 month of exclusive breastfeeding), mode of delivery (vaginal birth/caesarean), mother's level of education (from 0 - early childhood education to 8-doctoral or equivalent, in accordance with the International Standard Classification of Education, number of siblings, living environment (rural or urban), type of care (day care or individual) and child-feeding behaviour (age of onset of food diversification). The effect of prebiotics supplementation on $\mathrm{AD}$ at 1year will be tested in the following subgroups: (1) breastfeeding and (2) mode of delivery. For each of the subgroup analyses, the same statistical model as described for the main analysis will be used, integrating the interaction terms: breastfeeding *treatment; mode of delivery*treatment. In order to take into account the multiplicity of tests, an alpha risk adjustment by the Holm method will be applied.

Severity of $\mathrm{AD}$ and quality of life will be analysed using mixed models with the parental history of atopy as fixed effect and the centre as random effect. Sensitisation status to common allergens will be described in each group by frequency and percentage.

Tolerance will be captured by the analysis of the participants' diary. Each item (bloating, stomach aches, diarrhoea, flatulence) will be categorised on a monthly basis with a 4-point scale from absent (0) to severe (3) by the participants. Tolerance outcomes will be described in each group by frequency and percentage.

The per-protocol population will be defined as participants exposed to more than $75 \%$ of the expected prebiotics doses and with at least the 1-year outcome available.

\section{Recruitment}

Women will be informed about the trial by research staff during the telephone call regarding their routine antenatal follow-up appointments (taking place before 20-week gestation) at one of the participating centres. Pregnant women will also be informed about the trial by approved advertising material which will be circulated in hard copy. A participant information sheet describing the goal of the study, the procedures to be followed and the risks and benefits of participation will be provided to interested women. Following a screening process to ensure eligibility criteria are met, the investigator will conduct the informed consent discussion, check that information provided is understood and answer any questions about the study. A record of all women screened and their enrolment status will be maintained. Participants will be informed of their right to withdraw involvement in the trial at any time and the reason for withdrawal will be recorded where possible.

\section{Confidentiality and access to data}

In accordance with legislative provisions (articles L.1121-3 and R.5121-13 of the French Public Health Code), individuals charged with the quality control of the biomedical study who have access to confidential data will take all necessary precautions to ensure this confidentiality is maintained, particularly for data pertaining to the intervention, the investigational studies, the study participants and the results obtained.

\section{ETHICS AND DISSEMINATION}

\section{Research ethics approval}

The sponsor (Nantes University Hospital) and the investigators undertake to conduct this study in compliance 
with French law no 2004-806 of 9th August 2004 and following Good Clinical Practice and the Helsinki Declaration (Ethical Principles for Medical Research involving Human Subjects, Tokyo 2004). The study will be conducted in compliance with the current approved version of the protocol. Any change to the protocol document or informed consent form that affects the scientific intent, study design, patient safety or may affect a participant's willingness to continue participation in the study will be considered a major amendment. All such amendments will be submitted to the 'Comité de protection des Personnes SOOM III’ for approval.

\section{Dissemination}

Study findings will be submitted for peer-reviewed publication and for presentation at appropriate local and international conferences. In addition, the results of the study will be disseminated to participants in the form of a one-page lay summary.

\section{Author affiliations}

${ }^{1}$ UMR PhAN, INRA Centre Angers-Nantes, Nantes, Pays de la Loire, France

${ }^{2}$ Department of Dermatology, Centre Hospitalier Universitaire de Nantes, Nantes,

Pays de la Loire, France

${ }^{3}$ UMR PhAN, Centre Hospitalier Universitaire de Nantes, Nantes, Pays de la Loire, France

${ }^{4}$ CIC FEA, Centre Hospitalier Universitaire de Nantes, Nantes, Pays de la Loire, France

${ }^{5}$ Obstetrics and Gynecology Department, Centre Hospitalier Universitaire de Nantes, Nantes, Pays de la Loire, France

${ }^{6}$ Department of Clinical Research, Centre Hospitalier Universitaire de Nantes,

Nantes, Pays de la Loire, France

${ }^{7}$ Platform Methodology and Biostatistics, Centre Hospitalier Departemental Vendee, La Roche-sur-Yon, Pays de la Loire, France

${ }^{8}$ HUGOPEREN, Centre Hospitalier Universitaire de Nantes, Nantes, Pays de la Loire, France

${ }^{9}$ Department of Dermatology, Centre Hospitalier Regional Universitaire de Tours,

Tours, Centre, France

${ }^{10}$ Clinical Investigation Center-INSERM 1415, Centre Hospitalier Regional Universitaire de Tours, Tours, Centre, France

${ }^{11}$ Department of Obstetrics, Gynecology and Fetal Medicine, Centre Hospitalier

Regional Universitaire de Tours, Tours, France

${ }^{12}$ Maternité Olympe de Gouges, Hopital Bretonneau, Tours, Centre, France

${ }^{13}$ Department of Dermatology, Centre Hospitalier Universitaire de Rennes, Rennes,

Bretagne, France

${ }^{14}$ EA 7449 REPERES Pharmacoepidemiology and Health Services Research,

Universite de Rennes 1, Rennes, Bretagne, France

${ }^{15}$ Obstetrics and Gynecology Department, Centre Hospitalier Universitaire de Rennes, Rennes, Bretagne, France

${ }^{16}$ Department of Pharmacy, Centre Hospitalier Universitaire de Nantes, Nantes, UK

${ }^{17}$ Centre of Evidence Based Dermatology, University of Nottingham School of Medicine, Nottingham, UK

${ }^{18}$ BIA UR1268, INRA Centre Angers-Nantes, Nantes, Pays de la Loire, France

${ }^{19}$ Department of Dermatology, Centre Hospitalier Universitaire de Nantes, Nantes,

Pays de la Loire, France

Contributors MBod, SB, and HA contributed to the concept idea, the design and the development of the protocol. CC performed the feasibility study. BK, VB, MBoi, VD, NW, EF-P, LP, DR, AM, MT-S, FP, CD and LL contributed to the design and development of the study protocol. SB and NKR drafted the manuscript. All authors critically revised the protocol for intellectual content and approved the final version to be published.

Funding The PREGRALL study is supported by a grant from the French Ministry of Health (PHRCI 2015, No API15/N/048). The CIMMAP study is supported by a grant from the "Agence nationale de la Recherche" (ANR) DS04 ; Vie, santé et bien-être - 2017.
Competing interests None declared.

Patient consent for publication Obtained.

Ethics approval The protocol was approved on 27 April 2017 by the 'Comité de protection des Personnes SOOM III'.

Provenance and peer review Not commissioned; externally peer reviewed.

Open access This is an open access article distributed in accordance with the Creative Commons Attribution Non Commercial (CC BY-NC 4.0) license, which permits others to distribute, remix, adapt, build upon this work non-commercially, and license their derivative works on different terms, provided the original work is properly cited, appropriate credit is given, any changes made indicated, and the use is non-commercial. See: http://creativecommons.org/licenses/by-nc/4.0/.

\section{REFERENCES}

1. Pawankar R. Allergic diseases and asthma: a global public health concern and a call to action. World Allergy Organ J 2014;7:1-3.

2. DaVeiga SP. Epidemiology of atopic dermatitis: a review. Allergy Asthma Proc 2012;33:227-34.

3. Weidinger S, Beck LA, Bieber T, et al. Atopic dermatitis. Nat Rev Dis Primers 2018;4:1):1.

4. Nylund L, Nermes M, Isolauri E, et al. Severity of atopic disease inversely correlates with intestinal microbiota diversity and butyrateproducing bacteria. Allergy 2015;70:241-4.

5. Werfel T, Allam JP, Biedermann T, et al. Cellular and molecular immunologic mechanisms in patients with atopic dermatitis. J Allergy Clin Immunol 2016;138:336-49.

6. Pfefferle PI, Büchele G, Blümer N, et al. Cord blood cytokines are modulated by maternal farming activities and consumption of farm dairy products during pregnancy: the PASTURE Study. J Allergy Clin Immunol 2010;125-108-15.

7. Jiménez E, Fernández L, Marín ML, et al. Isolation of commensal bacteria from umbilical cord blood of healthy neonates born by cesarean section. Curr Microbiol 2005;51:270-4.

8. Gourbeyre P, Desbuards N, Grémy G, et al. Exposure to a galactooligosaccharides/inulin prebiotic mix at different developmental time points differentially modulates immune responses in mice. J Agric Food Chem 2012;60:11942-51.

9. Gourbeyre P, Desbuards N, Grémy G, et al. Perinatal and postweaning exposure to galactooligosaccharides/inulin prebiotics induced biomarkers linked to tolerance mechanism in a mouse model of strong allergic sensitization. J Agric Food Chem 2013;61:6311-20.

10. Bouchaud G, Castan L, Chesné J, et al. Maternal exposure to GOS/ inulin mixture prevents food allergies and promotes tolerance in offspring in mice. Allergy 2016;71:68-76.

11. Fujiwara R, Takemura N, Watanabe J, et al. Maternal consumption of fructo-oligosaccharide diminishes the severity of skin inflammation in offspring of NC/Nga mice. Br J Nutr 2010;103-530-8.

12. Hogenkamp A, Thijssen S, van Vlies N, et al. Supplementing pregnant mice with a specific mixture of nondigestible oligosaccharides reduces symptoms of allergic asthma in male offspring. J Nutr 2015;145-640-6.

13. Grüber C, van Stuijvenberg M, Mosca F, et al. Reduced occurrence of early atopic dermatitis because of immunoactive prebiotics among low-atopy-risk infants. J Allergy Clin Immunol 2010;126:791-7.

14. Grüber C, van Stuivenberg M, Mosca F, et al. Immunoactive prebiotics transiently prevent occurrence of early atopic dermatitis among low-atopy-risk infants. J Allergy Clin Immunol 2015;136:1696-8.

15. Cuello-Garcia C, Fiocchi A, Pawankar R, et al. Prebiotics for the prevention of allergies: A systematic review and meta-analysis of randomized controlled trials. Clin Exp Allergy 2017;47:1468-77.

16. Osborn DA, Sinn JKH. Cochrane Neonatal Group. Prebiotics in infants for prevention of allergy. Cochrane Database Syst Rev 2013;126:Cd006474.

17. Pedersen A, Sandström B, Van Amelsvoort JM. The effect of ingestion of inulin on blood lipids and gastrointestinal symptoms in healthy females. Br J Nutr 1997;78:215-22.

18. Simpson EL, Keck LE, Chalmers JR, et al. How should an incident case of atopic dermatitis be defined? A systematic review of primary prevention studies. J Allergy Clin Immunol 2012;130:137-44.

19. Asher MI, Keil U, Anderson HR, et al. International Study of Asthma and Allergies in Childhood (ISAAC): rationale and methods. Eur Respir J 1995;8:483-91.

20. Kunz B, Oranje AP, Labrèze $L$, et al. Clinical validation and guidelines for the SCORAD index: consensus report of the European Task Force on Atopic Dermatitis. Dermatology 1997;195:10-19.

21. Charman CR, Venn AJ, Williams HC. The patient-oriented eczema measure: development and initial validation of a new tool for 
measuring atopic eczema severity from the patients' perspective. Arch Dermatol 2004;140:1513-9.

22. Basra MK, Sue-Ho R, Finlay AY. The Family Dermatology Life Quality Index: measuring the secondary impact of skin disease. $\mathrm{Br} J$ Dermatol 2007;156:528-38.
23. Heinzerling L, Mari A, Bergmann KC, et al. The skin prick test European standards. Clin Transl Allergy 2013;3:3.

24. Wadonda-Kabondo N, Sterne JA, Golding J, et al. Association of parental eczema, hayfever, and asthma with atopic dermatitis in infancy: birth cohort study. Arch Dis Child 2004;89-917-21. 